\title{
Nonlinear plasmonic switching in graphene waveguide loaded with semiconductor nanowire
}

\author{
Alexander Shesterikov, and Alexei Prokhorov* \\ Vladimir State University named after A. G. and N. G. Stoletovs, Department of Physics and Applied \\ Mathematics, 600000 Vladimir, Russia
}

\begin{abstract}
A novel graphene waveguide loaded with semiconductor nanowire for control of surface plasmon-polariton propagation has been developed and simulated.
\end{abstract}

In this work we study the effect of nonlinear transmittance switching for surface plasmonpolariton (SPP) propagating along the graphene waveguide integrated with a stub nanoresonator loaded with a core-shell nanowire (NW), see Fig. 1a. For this purpose, the geometry of the system was chosen to realize the effective SPP-NW coupling [1]. We use the stub nanoresonator loaded with NW since such type of resonator can provide narrowband wave selection. To realize the switching effect, we propose to use the combination of two pump/signal SPP modes, which provide the Ladder-type scheme of SPP-NW interaction (see Fig. 1b). The pump SPP is utilized to excite the interband transition in NW and to provide the necessary electron population in the conduction band.
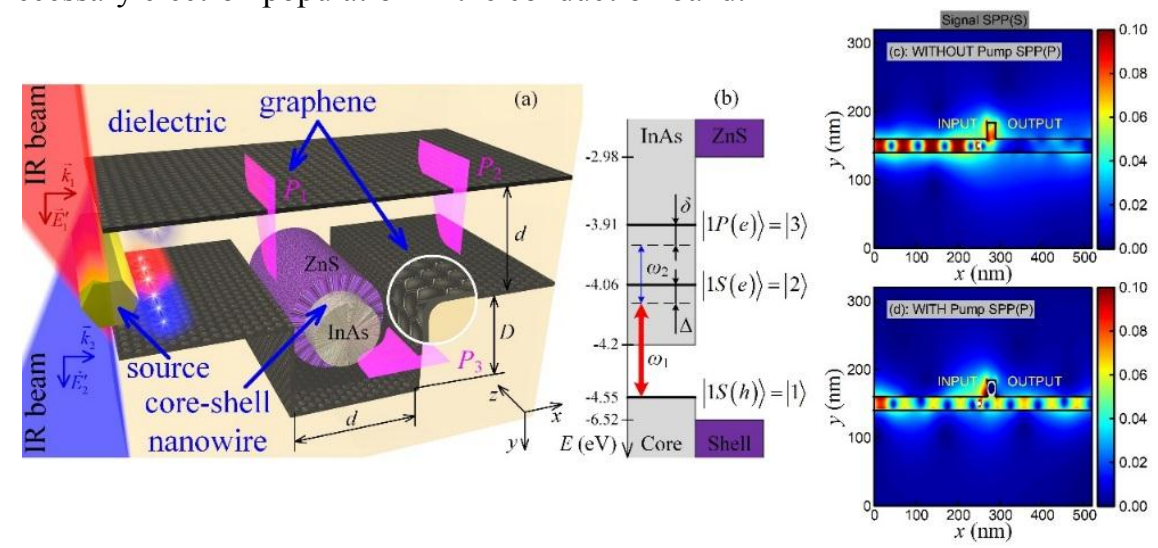

Fig. 1. (a) The model of plasmonic waveguide integrated with stub nanoresonator based on the pair of graphene sheets and core-shell NW; (b) the Ladder-type interaction scheme of two SPP modes and InAs/ZnS NW. The summarized electric field distribution of signal SPP in the device (c) without pump SPP and (d) with pump SPP. The black lines correspond to the graphene waveguide with stub nanoresonator and circled white line depicts the NW.

\footnotetext{
*Corresponding author: avprokhorov33@mail.ru
} 
The signal SPP is tuned to the intraband transition in the conduction band and get a controllable phase modulation due to interaction with NW. Initially, the resonator is tuned to the condition of minimum transmittance for signal SPP (about 7\%, see Fig. 1c) in the absence of pump SPP. The presence of pump SPP in the circuit leads to additional $\pi$ radians nonlinear phase shift of signal SPP, under which a change in the SPP pattern from destructive to constructive interference in nanoresonator occurs. Thus, when the pump SPP is on, the transmittance of the signal SPP reaches up to $93 \%$, see Fig. 1d.

We test the switching of signal SPP at $\lambda_{2}=8 \mu \mathrm{m}$ wavelength using pump SPP at $\lambda_{1}=2.56$ $\mu \mathrm{m}$ wavelength in the system with $\mathrm{InAs} / \mathrm{ZnS}$ core-shell $\mathrm{NW}$ (10 $\mathrm{nm}$ radius) loaded into graphene stub with width $d=22 \mathrm{~nm}$ [2]. We choose such graphene parameters and interlayer distance to provide a strong coupling between signal SPPs localized on parallel graphene sheets. Using full-wave electromagnetic simulation for SPPs, the complete agreement of numerical results with analytical solutions is demonstrated. As a result, we have proposed the model of all-plasmonic graphene transistor, which can provide the switching effect for SPPs in mid-infrared range accompanied by very narrow resonance lines. We have shown mutually reversible switching effect for such a device and determined its nominal clock frequency of about $100 \mathrm{GHz}$. The large-scale integration chips can be realized by graphenemetal hybrid creation technology [3].

This work was supported by the Foundation for Assistance to Small Innovative Enterprises (Agreement 14287GU/2019, UMNIK), by the Russian Foundation for Basic Research (project no. 17-42330001_r_a).

\section{References}

1. E. Bermúdez-Ureña, C. Gonzalez-Ballestero, M. Geiselmann, R. Marty, I.P. Radko, T. Holmgaard, Yu. Alaverdyan, E. Moreno, F.J. García-Vidal, S.I. Bozhevolnyi, R. Quidant, Nat. Commun. 6, 7883 (2015).

2. M.Yu. Gubin, A.Yu. Leksin, A.V. Shesterikov, A.V. Prokhorov, arXiv:1812.04487v2 [cond-mat.mes-hall] (2019)

3. V.S. Volkov, D.I. Yakubovsky, Y.V. Stebunov, R.V. Kirtaev, K.V. Voronin, A.V. Arsenin, J. Phys. Conf. Ser. 1092, 012161 (2018) 